\title{
Missed femur fracture in pediatric trauma patient
}

\begin{abstract}
Isolated paediatric femoral fracture is common and can be missed due to many Factors such as patient related, clinical, technical and radiological error. Careful listening to patient complain and examination along with careful look up and emphasising in radiological evaluation used for diagnosis. Prophylactic bed rest or hip brace is an option in such condition for short period and callus might show up.
\end{abstract}

Volume II Issue 4 - 2019

\author{
Ahmad N Boeisa, Mohammed S Alhassan, Ali \\ ALMuslami \\ Department of Orthopedic, King Fahad Hospital, Saudi Arabia
}

Correspondence: Ahmad N Boeisa, Department of Orthopedic, King Fahad Hospital, Saudi Arabia, Email ahmadboeisa@gmail.com

Received: July 20, 2019 | Published: July 30, 2019

\section{Introduction}

Isolated pediatric femoral shaft fractures occur at an annual rate of 19 per 100,000 and represent the most frequent pediatric orthopaedic injury requiring hospitalization. ${ }^{1,2}$ The treatment of these fractures is dictated by various factors. The orthopaedic surgeon caring for these fractures must take into account the patients age, weight, family circumstances, and fracture pattern to determine the best treatment options. The cost for these various treatment options can also come into consideration. ${ }^{1-3}$

A recent annual review of paediatric trauma patients from our institution found five patients $(1.6 \%)$ with missed fractures and other musculoskeletal (MSK) injuries. ${ }^{4}$ Several previous studies have noted a high incidence of missed or occult MSK injuries in both paediatric and adult patients with head injuries and/or multiple trauma

numerous factors in the failure to detect MSK injuries during the initial resuscitation and examination. Soundappan noted that more than one factor was responsible for missed injury in $30 \%$ of patients.

\section{Factors divided}
a. Patient related
b. Clinical
c. Technical
d. Radiological
e. Admission to inappropriate service

\section{Case report}

Our case is 4 years old boy brought to trauma pay by red crescent as victim of motor vehicle accident frontal impaction. On arrival ATLS primary and secondary surveys applied and patient found to be free except for left thigh pain. Patient re-examined by different ranked orthopaedic surgeons and found to have pain mainly in distal thigh, inspection revel no signs of local trauma, palpation resulted in tenderness over distal thigh, patient has full painful range of motion of hip and knee joint. Patient was able to stand over his left lower limb but can't proceed with walking. Distal neurovascular exam was normal.

Patient discharged with diagnosis of contusion. Patient visited emergency department in two consecutive days with the same complain of left thigh pain at even at rest and inability to bear weight where he were evaluated by different orthopaedic surgeons and has same impression of contusion. At third visit patient brought by family with deformed left thigh after unattended incident at home.

\section{Investigations}

As part of primary survey patient's chest and pelvis X-rays done at trauma bay shows no abnormality. AP and lateral X-rays of left femur showing hip and knee joints were reviewed by different ranked orthopaedics surgeons with agreement of normal x-ray impression (Figure $1 \& 2$ ). The following visits for the same complain has different x-rays with same impression of normal x-ray (Figure 3). Except for the one at last visit with the clinical evidence of deformity, patient has diaphysial femur fracture (Figure 4 ).

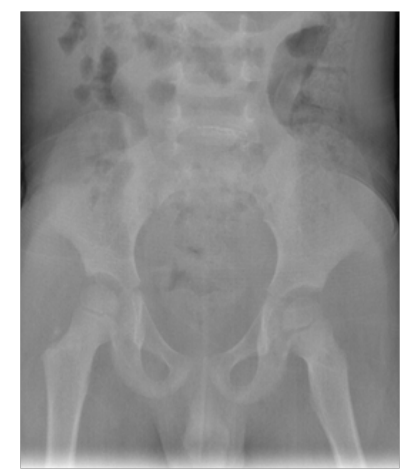

Figure I Anterior, posterior pelvis x-ray showing left hip
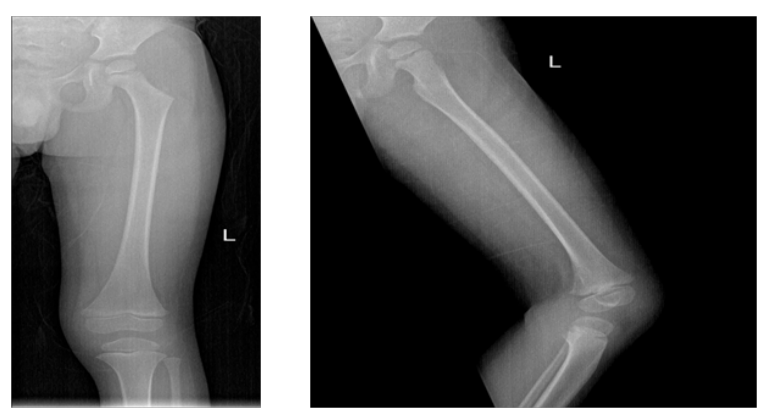

Figure 2 Posterior, anterior and lateral x-ray of left femur showing knee. 

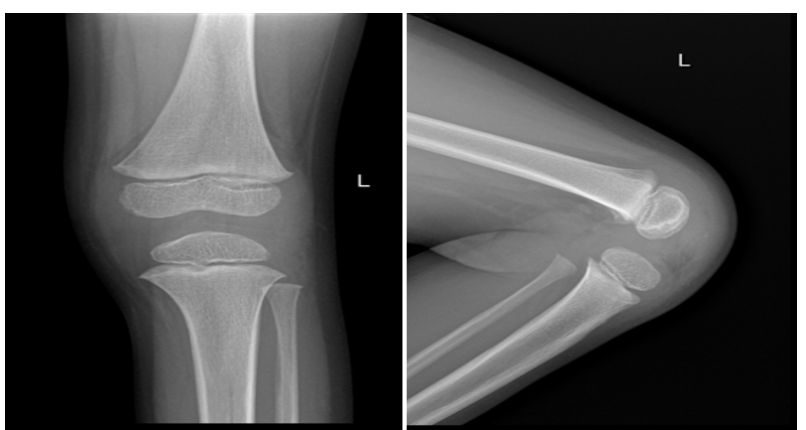

Figure 3 Different x-rays with same impression of normal x-ray.

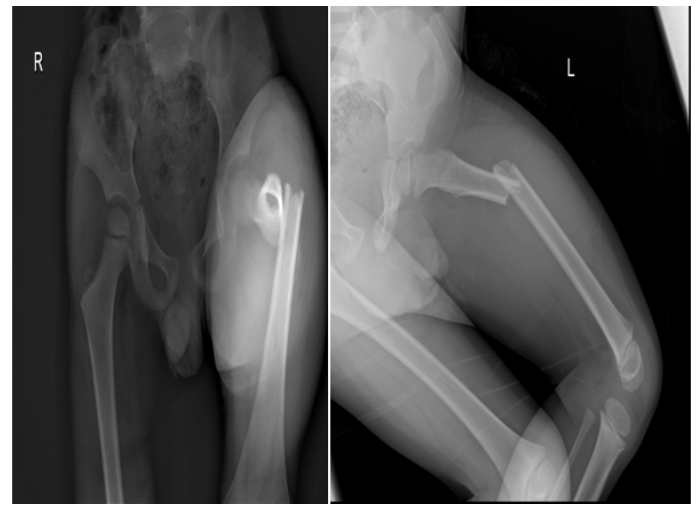

Figure $4 \times$ ray showing diaphysial femur fracture.
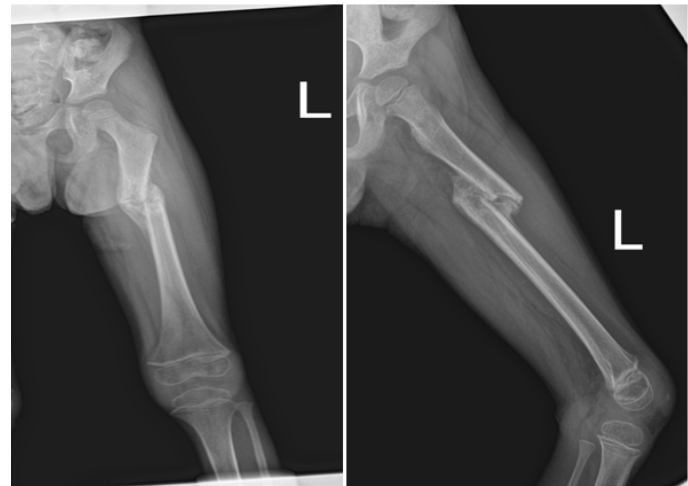

Figure $5 \times$ ray of healed femur fracture.

\section{Differential diagnosis}

The repetitive visits with the same complain to ER increase suspicion of injury presence.

Compartment syndrome and referred pain were excluded by physical examination and final diagnosis made as contusion.

\section{Treatment}

Patient treated primarily by reassurance, analgesia and instructions of rest and to revisit ER if pain progressed.
After evidence of fracture patient treated with immediate hip spica cast.

\section{Outcome and follow-up}

Multiple OPD visits shows well aligned and healed femur fracture within expected time frame (Figure 5).

\section{Discussion}

Reviewing literature shows no reported similar cases.

This patient has been missed of diagnosis with:

a. Liable patient related factors as he was fully conscious and reliable with repeating his complain of thigh pain

b. clinical factor was also eliminated as the patient has been examined thoroughly by different doctors in different setups and times

c. Technical factors has been eliminated also as the patient has no obstacles in his clinical condition to cover his injury specifically in his limb

d. Radiological factors has deficient of only AP view in the initial visit

But in second visit proper AP and lateral views which interpreted multiple times without findings

Finally patient hasn't been admitted under any speciality and discharged.

\section{Learning points/take home messages}

a. Careful listen to patients complain and overlook it as per described protocols

b. Suggestion of aggressive radiological investigation with repeated visit to ER

c. Prophylactic bed rest or hip brace is an option in such condition for short period and callus might show up

\section{Acknowledgments}

None.

\section{Conflicts of interest}

The authors declare there is no conflict of interest.

\section{References}

1. Loder RT, O'Donnell PW, Feinberg JR. Epidemiology and mechanisms of femur fractures in children. J Pediatr Orthop. 2006;26(5):561-566.

2. Hinton RY, Lincoln A, Crockett MM, et al. Fractures of the femoral shaft in children: incidence, mechanisms, and sociodemographic risk factors. $J$ Bone Joint Surg Am. 1999;81(4):500-509.

3. Flynn JM, Schwend RM. Management of pediatric femoral shaft fractures. J Am Acad Orthop Surg. 2004;12(5):347-349.

4. Segal LS, Shrader MW. Missed fractures in paediatric trauma patients. Acta Orthop Belg. 2013;79(6):608-615. 